Discrete Comput Geom 35:457-471 (2006)

DOI: $10.1007 / \mathrm{s} 00454-005-1204-0$

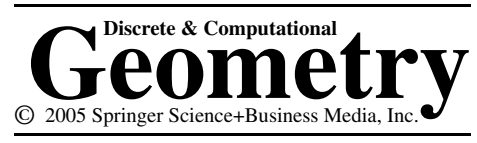

\title{
On the Bounded Complex of an Affine Oriented Matroid
}

\author{
Xun Dong \\ Department of Mathematics, University of Miami, \\ Coral Gables, FL 33124, USA \\ xundong@math.miami.edu
}

\begin{abstract}
We prove that the bounded complex of an affine oriented matroid is pure and collapsible. We also generalize Zaslavsky's central decomposition theorem for hyperplane arrangements to affine oriented matroids.
\end{abstract}

\section{Introduction}

While arrangements of hyperplanes in $\mathbb{R}^{d}$ arise as fundamental objects in various mathematical theories, they have also been studied for a long time by discrete geometers. In Zaslavsky's classic 1975 treatise of hyperplane arrangements [8], he studies how the hyperplanes partition the space and proves many beautiful face enumeration formulas. Zaslavsky [8] also develops a theory of bounded faces. Let $\mathcal{A}=\left\{H_{1}, \ldots, H_{n}\right\}$ be a hyperplane arrangement in $\mathbb{R}^{d}$, where $H_{i}=\left\{\mathbf{x} \in \mathbb{R}^{d}: \sum_{j=1}^{d} a_{i j} x_{j}=b_{j}\right\}$. These affine hyperplanes partition the space into faces. The bounded faces are those bounded in the usual metric sense. The collection of all bounded faces is a polyhedral complex called the bounded complex of $\mathcal{A}$.

Some of Zaslavsky's formulas were independently discovered by Las Vergnas [5] for the more general oriented matroids. Zaslavsky's other formulas and theory were later generalized to oriented matroids as well. Oriented matroids have a topological model as arrangements of pseudo-hyperplanes, each obtained from a flat hyperplane by tame topological deformation. In this model the hyperplane arrangements correspond to realizable affine oriented matroids. Since we can still talk about faces and a metric in a pseudo-hyperplane arrangement, the bounded complex of an affine oriented matroid can be defined as the regular cell complex consisting of all bounded faces. At first glance, the bounded complex of an affine oriented matroid seems to be a simple object. However, there are still unanswered questions about its topology. In this paper we address two such questions which are described in the next two paragraphs. 
Let $\mathcal{A}$ be a hyperplane arrangement in $\mathbb{R}^{d}$. Let $\Gamma$ denote its bounded complex. A basic question about $\Gamma$ is whether it is pure, i.e., whether all maximal faces of $\Gamma$ have the same dimension. This question was listed as an open problem in Stanley's PCMI lecture notes [7]. A closer examination of [8] reveals that an affirmative answer of the question is provided by Zaslavsky's central decomposition theorem (see Corollary 9.1 of [8]). The same question can be asked for the bounded complex of an affine oriented matroid. However, Zaslavsky's method only works for hyperplane arrangements and does not generalize to the nonrealizable affine oriented matroids. In this paper we use the axioms of covectors to prove that the bounded complex is pure for any affine oriented matroid. Our method also generalizes Zaslavsky's central decomposition theorem of hyperplane arrangements to affine oriented matroids.

Another question is related to the homotopy type of the bounded complex. Zaslavsky [8] conjectured in 1975 that the bounded complex of a hyperplane arrangement is contractible. This was proved by Ziegler [9] in 1988. His proof does not generalize to the nonrealizable case. The generalized conjecture, that the bounded complex of an affine oriented matroid is contractible, was proved by Björner and Ziegler [1]. Stanley asked whether the bounded complex is collapsible (personal communication). A collapsible complex is contractible, but not vice versa. In this paper we use the axioms of covectors and Forman's discrete Morse theory to prove that the bounded complex of an affine oriented matroid is indeed collapsible. In a subsequent paper, the collapsibility will be used to show that the bounded complex of a uniform or simplicial affine oriented matroid is homeomorphic to a ball.

The paper is organized as follows. In Section 2 we provide a new proof of Zaslavsky's central decomposition theorem for hyperplane arrangements. One of the reasons we present this proof is that it provides some geometric insights into our proofs in Section 3, where we show that the bounded complex of an affine oriented matroid is pure. In Section 4 we develop a theory of parallel connection of oriented matroids, which turns out to be the right generalization of the direct sum of hyperplane arrangements. In Section 5 we formulate a central decomposition theorem for affine oriented matroids in terms of parallel connection. In Section 6 we prove that the bounded complex of an affine oriented matroid is collapsible.

\section{Hyperplane Arrangements}

In this section we prove a theorem which is essentially Zaslavsky's central decomposition theorem. Unlike Zaslavsky's original proof, our proof does not depend on his enumeration formula for the number of bounded faces. Let $\mathcal{A}=\left\{H_{1}, \ldots, H_{n}\right\}$ be a hyperplane arrangement in $\mathbb{R}^{d}$, where $H_{i}=\left\{\mathbf{x} \in \mathbb{R}^{d}: \sum_{j=1}^{d} a_{i j} x_{j}=b_{j}\right\}$. We assume that $\mathcal{A}$ is essential, that is, its bounded complex $\Gamma$ is nonempty. For a subset $S \subseteq \mathbb{R}^{d}$, the affine span of $S$ is the minimal affine subspace of $\mathbb{R}^{d}$ that contains $S$. For affine subspaces $V$ and $L$ in $\mathbb{R}^{d}$, we say that $L$ is an affine complement of $V$ if $L \cap V$ is a point and $\operatorname{dim} L+\operatorname{dim} V=d$. We say that a hyperplane $H$ is parallel to $L$ if $H \cap L=L$ or $\emptyset$. The following theorem is equivalent to Theorem $\mathrm{E}$ of [8]. 
Theorem 2.1. Let $\tau$ be a maximal bounded face of $\mathcal{A}$. Let $V$ be the affine span of $\tau$. Then

(1) one can find an affine complement $L$ of $V$ in $\mathbb{R}^{n}$, and a partition of the hyperplanes into two sets $\mathcal{A}_{1}$ and $\mathcal{A}_{2}$, such that the intersection of hyperplanes in $\mathcal{A}_{1}$ is $V$ and every hyperplane in $\mathcal{A}_{2}$ is parallel to $L$;

(2) all bounded faces of $\mathcal{A}$ are contained in $V$.

Corollary 2.2. The bounded complex $\Gamma$ of $\mathcal{A}$ is pure.

Proof. Since every maximal bounded face has the same affine span, they must all have the same dimension.

Proof of the Theorem. If $\operatorname{dim} \tau=d$, then the affine span of $\tau$ is $V=\mathbb{R}^{d}$. For the first part of the theorem we let $L$ be a point that is not in any of the hyperplanes, and partition $\mathcal{A}$ into $\mathcal{A}_{1}=\emptyset$ and $\mathcal{A}_{2}=\mathcal{A}$.

Now suppose that $\operatorname{dim} \tau<d$. Without loss of generality we assume that $\mathbf{0}$ is in the relative interior of $\tau$. Then its affine span $V$ is in fact a linear subspace. Let $\mathcal{A}_{1}=$ $\left\{H_{1}, \ldots, H_{k}\right\} \subseteq \mathcal{A}$ be the set of hyperplanes that contain $\tau$, so that $H_{1} \cap \cdots \cap H_{k}=V$. Let $\left\{W_{1}, \ldots, W_{m}\right\}$ be all possible intersections of hyperplanes in $\mathcal{A}$ such that $V \subset W_{i}$ and $\operatorname{dim} V+1=\operatorname{dim} W_{i}$ for all $i$. In other words, the $W_{i}$ 's are those flats covered by $V$ in the intersection poset $L(\mathcal{A})$.

For a fixed $i$, we consider the lower-dimensional hyperplane arrangement $\mathcal{A}_{W_{i}}=$ $\left\{H \cap W_{i}: H \in \mathcal{A}\right\}$, the restriction of $\mathcal{A}$ to $W_{i}$. Note that the faces of $\mathcal{A}_{W_{i}}$ are exactly those faces of $\mathcal{A}$ contained in $W_{i}$, therefore $\tau$ is still a maximal bounded face in $\mathcal{A}_{W_{i}}$. The codimension of $\tau$ in $W_{i}$ is one, thus $\tau$ is in the boundaries of two unbounded regions $X$ and $Y$ which lie in the two sides of $V$ in $W_{i}$.

Since $X$ and $Y$ are unbounded, we can find points $\mathbf{x} \in X$ and $\mathbf{y} \in Y$ such that the rays $\mathbb{R}_{+} \mathbf{x}=\{a \mathbf{x}: a>0\}$ and $\mathbb{R}_{+} \mathbf{y}$ do not hit any new hyperplanes. In other words, they do not intersect any hyperplanes other than those that contain the whole rays. We claim that $\mathbb{R}_{+} \mathbf{x}$ and $\mathbb{R}_{+} \mathbf{y}$ are on the same line, i.e., $\mathbf{y}=-a \mathbf{x}$ for some $a>0$. If $\operatorname{dim} V=0$ then this is clear. If $\operatorname{dim} V>0$ and $\mathbb{R}_{+} \mathbf{x}, \mathbb{R}_{+} \mathbf{y}$ are not on the same line, then we restrict $\mathcal{A}$ to the two-dimensional subspace $P$ spanned by $\mathbb{R}_{+} \mathbf{x}$ and $\mathbb{R}_{+} \mathbf{y}$. Note that $\tau \cap P$, $X \cap P$ and $Y \cap P$ are faces in this restriction $\mathcal{A}_{P}$. We also have $\operatorname{dim}(\tau \cap P)=1$, because $\operatorname{dim}(V \cap P)=\operatorname{dim} V+\operatorname{dim} P-\operatorname{dim} W_{i}=1$ and $\mathbf{0} \in V \cap P$ is in the relative interior of $\tau$. The faces $X \cap P$ and $Y \cap P$ are still unbounded, as they contain the rays $\mathbb{R}_{+} \mathbf{x}$ and $\mathbb{R}_{+} \mathbf{y}$, respectively. However, this is only possible when the lines defining the boundaries of $X \cap P$ and $Y \cap P$ (except $\tau \cap P$ ) are all parallel to each other, but then either $\mathbb{R}_{+} \mathbf{x}$ or $\mathbb{R}_{+} \mathbf{y}$ will intersect the boundary, since they are not on the same line. This is a contradiction. Therefore $\mathbb{R}_{+} \mathbf{x}$ and $\mathbb{R}_{+} \mathbf{y}$ must be on the same line. We have now found a line $L_{i}:=\mathbb{R} \mathbf{x} \subset W_{i}$ which does not intersect any of the hyperplanes in $\mathcal{A}_{2}=\mathcal{A} \backslash\left\{H_{1}, \ldots, H_{k}\right\}$. Let $L$ be the linear span of the lines $L_{i}, 1 \leq i \leq m$. Then $L \cap H=\emptyset$ for every $H \in \mathcal{A}_{2}$.

Note that $L_{i}+V=W_{i}$ and $W_{1}+\cdots+W_{m}=\mathbb{R}^{n}$, therefore $L+V=\mathbb{R}^{n}$. If $L \cap V$ contains a nonzero point $\mathbf{x}$, then $\mathbb{R} \mathbf{x} \subseteq V$ implies that $\mathbb{R} \mathbf{x}$ intersects a hyperplane defining 
the boundary of $\tau$ in $V$. On the other hand $\mathbb{R} \mathbf{x} \subseteq L$ implies $\mathbb{R} \mathbf{x}$ should not intersect such a hyperplane. This is a contradiction. Hence $L \cap V=\{\mathbf{0}\}$.

It remains to show that all bounded faces are contained in $V$. To see this we take a face $\sigma$ not in $V$. Let $\mathbf{x}$ be a point in its relative interior, so $\mathbf{x} \in H$ implies that $\sigma \subseteq H$ for a hyperplane $H \in \mathcal{A}$. Then $\mathbf{x}=\mathbf{v}+\mathbf{l}$ for some $\mathbf{v} \in V$ and $\mathbf{l} \in L \backslash\{\mathbf{0}\}$. Let $H$ be a hyperplane in $\mathcal{A}$. If $\mathbf{x} \in H$, then the ray $R=\{\mathbf{v}+a \mathbf{l}: a \geq 1\}$ starting at $\mathbf{x}$ is also contained in $H$ : either $H$ is parallel to $\mathbb{R} \mathbf{l}$, in which case $R$ is parallel to $H$ and therefore contained in $H$; or $H$ contains $\mathbf{v}$, in which case $H$ contains two points on the line spanned by $R$ and therefore contains $R$. Hence $R$ is contained in the affine span of $\sigma$. If $\mathbf{x} \notin H$ then $R \cap H=\emptyset$ : either $H$ contains $\mathbf{v}$, in which case the ray in the opposite direction, $\{\mathbf{v}+a \mathbf{l}: a<1\}$, hits $H$; or $H$ is parallel to $\mathbb{R} \mathbf{l}$, in which case $R$ is also parallel to $H$ and therefore does not intersect $H$. This shows that $R$ does not hit any hyperplane defining the boundary of $\sigma$. Therefore the whole ray $R$ is contained in $\sigma$ and $\sigma$ is unbounded.

\section{Affine Oriented Matroids}

In this section we prove that the bounded complex of an affine oriented matroid is pure. Our proofs utilize the covector axioms, so we start with a quick review of the necessary definitions and terminology. The following notation is mostly taken from Section 4.1 of [2].

Let $E$ be a finite set and consider the sign vectors $X, Y \in\{+,-, 0\}^{E}$. The support of a vector $X$ is $\operatorname{sp}(X)=\left\{e \in E: X_{e} \neq 0\right\}$; its zero set is

$$
z(X)=E \backslash \operatorname{sp}(X)=\left\{e \in E: X_{e}=0\right\} .
$$

The opposite of a vector $X$ is $-X$, defined by

$$
(-X)_{e}=\left\{\begin{array}{lll}
-, & \text { if } & X_{e}=+ \\
+, & \text { if } & X_{e}=- \\
0, & \text { if } & X_{e}=0
\end{array}\right.
$$

The zero vector is 0 , with $0_{e}=0$ for all $e \in E$. The composition of two vectors $X$ and $Y$ is $X \circ Y$, defined by

$$
(X \circ Y)_{e}= \begin{cases}X_{e}, & \text { if } X_{e} \neq 0 \\ Y_{e}, & \text { otherwise }\end{cases}
$$

The separation set of $X$ and $Y$ is $S(X, Y)=\left\{e \in E: X_{e}=-Y_{e} \neq 0\right\}$. Notice that $X \circ Y=Y \circ X$ if and only if $S(X, Y)=\emptyset$, in which case we say that $X$ and $Y$ are conformal. We are now ready for the definition of oriented matroids in terms of covectors (see 4.1.1 of [2]).

Definition 3.1 (Covector Axioms). An oriented matroid is a pair $(E, \mathcal{L})$, where $E$ is a finite set and $\mathcal{L} \subseteq\{+,-, 0\}^{E}$ is the set of covectors satisfying:

(L0) $0 \in \mathcal{L}$;

(L1) $X \in \mathcal{L}$ implies that $-X \in \mathcal{L}$; 
(L2) $X, Y \in \mathcal{L}$ implies that $X \circ Y \in \mathcal{L}$;

(L3) if $X, Y \in \mathcal{L}$ and $e \in S(X, Y)$ then there exists $Z \in \mathcal{L}$ such that $Z_{e}=0$ and $Z_{f}=(X \circ Y)_{f}=(Y \circ X)_{f}$ for all $f \notin S(X, Y)$.

Let $\leq$ be the partial order on the set $\{+,-, 0\}$ defined by $0<+$ and $0<-$, with + and - incomparable. This induces a product partial order on $\{+,-, 0\}^{E}$. Thus $Y \leq X$ if and only if $Y_{e} \in\left\{0, X_{e}\right\}$ for all $e \in E$. As a subset of $\{+,-, 0\}^{E}$ the set of covectors $\mathcal{L}$ has an induced partial order with bottom element 0 . Let $\widehat{\mathcal{L}}$ denote the poset $\mathcal{L}$ with a top element $\hat{1}$ adjoined. Then $\widehat{\mathcal{L}}$ is a lattice called the big face lattice of $(E, \mathcal{L})$. The join in $\widehat{\mathcal{L}}$ of $X$ and $Y$ equals $X \circ Y=Y \circ X$ if $S(X, Y)=\emptyset$, and equals $\hat{1}$ otherwise.

An affine oriented matroid is a triple $(E, \mathcal{L}, g)$, where $(E, \mathcal{L})$ is an oriented matroid and $g \in E$ is a distinguished element which is not a loop. Recall that $g$ is a loop if $X_{g}=0$ for all $X \in \mathcal{L}$. We now define the bounded complex as in Definition 4.5.1 of [2]. For an affine oriented matroid $(E, \mathcal{L}, g)$ let

$$
\mathcal{L}^{+}=\left\{X \in \mathcal{L}: X_{g}=+\right\} \quad \text { and } \quad \widehat{\mathcal{L}}^{+}=\mathcal{L}^{+} \cup\{0, \hat{1}\} .
$$

With the induced order as a subset of $\widehat{\mathcal{L}}$, we call $\widehat{\mathcal{L}}^{+}$the affine face lattice of $(E, \mathcal{L}, g)$. The bounded complex of $(E, \mathcal{L}, g)$ is

$$
\mathcal{L}^{++}=\left\{X \in \mathcal{L}^{+}: \mathcal{L}_{\leq X} \subseteq \widehat{\mathcal{L}}^{+}\right\} .
$$

We freely use the following basic facts about the posets $\mathcal{L}, \widehat{\mathcal{L}}, \mathcal{L}^{+}, \widehat{\mathcal{L}}^{+}$and $\mathcal{L}^{++}$ throughout the paper:

(1) $\mathcal{L}$ is the face poset of a shellable regular cell decomposition of an $(r-1)$-sphere, where $r$ is the rank of the underlying matroid of $(E, \mathcal{L})$. In particular, $\widehat{\mathcal{L}}$ is graded of length $r+1$. (See Theorem 4.3.3 of [2].)

(2) $\mathcal{L}^{++} \cup\{0\}$ is an order ideal of $\mathcal{L}$. Therefore it is the face poset of a subcomplex of the $(r-1)$-sphere.

(3) $\mathcal{L}^{+}$is an order filter in $\mathcal{L}$. It is pure of length $r-1$, so the minimal covectors in $\mathcal{L}^{+}$are atoms in $\mathcal{L}$. (See Proposition 4.5.3 of [2].)

(4) The set $L=\{z(X): X \in \mathcal{L}\}$ is the collection of flats of the underlying matroid. The map $z: \mathcal{L} \rightarrow L$ is a cover-preserving, order-reversing surjection of $\mathcal{L}$ onto the geometric lattice $L$. In particular, this implies that if $\operatorname{sp}(X) \subset \operatorname{sp}(Y)$, then $\operatorname{rank}(X)<\operatorname{rank}(Y)$; if $\operatorname{sp}(X)=\operatorname{sp}(Y)$ then $\operatorname{rank}(X)=\operatorname{rank}(Y)$. (See Proposition 4.1 .13 of [2].)

(5) $\widehat{\mathcal{L}}$ is an atomic lattice, i.e., every covector is a join (composition) of atoms. In particular, all atoms below a fixed covector are conformal, so that composition is commutative. (See Proposition 3.7.2 of [2].)

(6) Let $[X, \hat{1}]$ be an interval in $\widehat{\mathcal{L}}$. Then there exists an oriented matroid $\left(E^{\prime}, \mathcal{L}^{\prime}\right)$ such that $[X, \hat{1}] \cong \widehat{\mathcal{L}}^{\prime}$. In particular, $[X, \hat{1}]$ is atomic. (See Proposition 4.1 .9 of $[2]$.)

(7) The maximal covectors are called topes. All topes have the same support - the set of all nonloop elements in $E$. Let $\mathcal{T}=\mathcal{T}(\mathcal{L})$ denote the set of topes. Then $\mathcal{T}$ determines $\mathcal{L}$ via $\mathcal{L}=\left\{X \in\{+,-, 0\}^{E}: X \circ T \in \mathcal{T}\right.$ for all $\left.T \in \mathcal{T}\right\}$. (See Theorem 4.2 .13 of [2].)

Aside from the usual notations, we need a new notation about sign vectors for the convenience of our discussion. Let $Y \in\{+,-, 0\}^{E}$ be a sign vector and let $F \subseteq E$. 
Then $Y_{F} \in\{+,-, 0\}^{E}$ is the sign vector defined by $\left(Y_{F}\right)_{e}=Y_{e}$ if $e \in F$, and $\left(Y_{F}\right)_{e}=0$ otherwise. Notice the difference between $\left.Y\right|_{F}$ and $Y_{F}$ : The former is the restriction of $Y$ to $F$; the latter is the restriction of $Y$ to $F$ in a sense required by the need for $Y_{F}$ to be defined on all of $E$. In particular, if $X, Y \in\{+,-, 0\}^{E}$ are sign vectors, then $Y_{z(X)}$ and $Y_{\mathrm{sp}(X)}$ are the sign vectors with

$$
\left(Y_{z(X)}\right)_{e}=\left\{\begin{array}{ll}
0, & \text { if } \quad e \in \mathrm{sp}(X), \\
Y_{e}, & \text { if } \quad e \in z(X) ;
\end{array} \text { and } \quad\left(Y_{\mathrm{sp}(X)}\right)_{e}=\left\{\begin{array}{lll}
Y_{e}, & \text { if } \quad e \in \mathrm{sp}(X), \\
0, & \text { if } \quad e \in z(X) .
\end{array}\right.\right.
$$

We now state a theorem that is analogous to our Theorem 2.1. The geometric idea behind its proof is the same as that of Theorem 2.1, only disguised in the language of covectors.

Theorem 3.2. Let $(E, \mathcal{L}, g)$ be an affine oriented matroid. Let $X \in \mathcal{L}^{++}$be a maximal covector in the bounded complex.

(1) For any covector $Y \in \mathcal{L}$, we have $Y_{z(X)} \in \mathcal{L}$ and $Y_{\mathrm{sp}(X)} \in \mathcal{L}$.

(2) If $W \in \mathcal{L}^{++}$then $\operatorname{sp}(W) \subseteq \operatorname{sp}(X)$.

Proof. (1) We first prove the special case when $X \lessdot Y$, i.e., the covector $Y$ covers $X$ in the poset $\mathcal{L}$. In this case $Y_{\operatorname{sp}(X)}=X \in \mathcal{L}$. We need to show that $Y_{z(X)} \in \mathcal{L}$ as well. Let $Y^{\prime}=X \circ(-Y)$. Then it is clear that $Y^{\prime}$ covers $X$. Since $X$ is maximal in the bounded complex $\mathcal{L}^{++}$, both $Y$ and $Y^{\prime}$ are not in $\mathcal{L}^{++}$. Therefore there exist nonzero covectors $Z, Z^{\prime} \in \mathcal{L} \backslash\{0\}$ such that $Z<Y, Z^{\prime}<Y^{\prime}$ and $Z_{g}=Z_{g}^{\prime}=0$. We now show that $Z=Y_{Z(X)}$. Note that $Z_{e} \leq Y_{e}=X_{e}$ for all $e \in \operatorname{sp}(X)$, and that $Z \not \leq X$ since $Z \notin \widehat{\mathcal{L}}^{+}$and $X \in \mathcal{L}^{++}$. Hence we have $X<X \circ Z \leq Y$. The fact that $Y$ covers $X$ then implies that $X \circ Z=Y$. In other words, $Z_{e}=Y_{e}$ if $e \in z(X)$. Similarly we can show that $Z_{e}^{\prime}=Y_{e}^{\prime}=-Y_{e}$ if $e \in z(X)$. Now take a fixed element $h \in S\left(Z, Z^{\prime}\right)=S\left(Y, Y^{\prime}\right) \subseteq z(X)$. By the Covector Axiom (L3) there exists $U \in \mathcal{L}$ such that $U_{h}=0$ and $U_{f}=\left(Z \circ Z^{\prime}\right)_{f}=\left(Z^{\prime} \circ Z\right)_{f}$ for all $f \notin S\left(Z, Z^{\prime}\right)$. We claim that $U<X$. Clearly $U_{e} \leq X_{e}$ if $e \in \operatorname{sp}(X)$, and $U \neq X$ since $U_{g}=0$. If $U_{e} \neq 0$ for some $e \in z(X)$ then we would have $X<X \circ U$. On the other hand since $\operatorname{sp}(X \circ U) \subset \operatorname{sp}(Y)$ we get $\operatorname{rank}(X \circ U)<\operatorname{rank}(Y)$, contradicting the fact that $Y$ covers $X$. Therefore $U<X$. This, together with the facts that $U_{g}=0$ and $X \in \mathcal{L}^{++}$, forces $U=0$. Hence $U_{e}=\left(Z \circ Z^{\prime}\right)_{e}=0$ for all $e \notin S\left(Z, Z^{\prime}\right)$. Consequently $Z_{e}=Z_{e}^{\prime}=0$ if $e \in \operatorname{sp}(X)$. We can now conclude that $Z=Y_{z(X)}$, and therefore $Y_{z(X)} \in \mathcal{L}$.

Next we prove the case when $X<Y$. In this case also $Y_{\mathrm{sp}(X)}=X \in \mathcal{L}$. To show that $Y_{z(X)} \in \mathcal{L}$, we consider the interval $[X, \hat{1}]$ in $\widehat{\mathcal{L}}$. Since this interval is an atomic lattice on its own, we can write $Y$ in the form $Y_{1} \circ \cdots \circ Y_{k}$ where $Y_{i}$ covers $X$ for all $i$. We have shown that $Z_{i}=\left(Y_{i}\right)_{z(X)}$ are covectors. Notice that $Y_{z(X)}=Z_{1} \circ \cdots \circ Z_{k}$. Therefore $Y_{z(X)}$ is a covector as well.

For the general case, we first prove that $Y_{z(X)} \in \mathcal{L}$. We consider the covector $W=$ $X \circ Y$. It is clear that $Y_{z(X)}=W_{z(X)}$ and $X \leq W$. If $X=W$, then $W_{z(X)}=0 \in \mathcal{L}$. If $X<W$ then $W_{z(X)} \in \mathcal{L}$ by the previous paragraph. It remains to show that $Y_{\operatorname{sp}(X)} \in \mathcal{L}$. We use induction on the size of $S\left(Y,-Y_{z(X)}\right)$. If $S\left(Y,-Y_{z(X)}\right)=\emptyset$ then $Y_{\operatorname{sp}(X)}=Y \in \mathcal{L}$. Otherwise let $h \in S\left(Y,-Y_{z(X)}\right)$. Then by the Covector Axiom (L3) there exists $Z \in \mathcal{L}$ 
such that $Z_{h}=0$ and $Z_{f}=\left(Y \circ\left(-Y_{z(X)}\right)\right)_{f}$ for all $f \notin S\left(Y,-Y_{z(X)}\right)$. It follows that $Z_{\mathrm{sp}(X)}=Y_{\mathrm{sp}(X)}$ and $S\left(Z,-Z_{z(X)}\right) \subset S\left(Y,-Y_{z(X)}\right)$. By induction $Z_{\mathrm{sp}(X)} \in \mathcal{L}$.

(2) For any given $W \in \mathcal{L}^{++}$, we have $W_{z(X)} \in \mathcal{L}$ by part (1). Thus $W_{z(X)} \in \mathcal{L}_{\leq W}^{++}$. Therefore $W_{z(X)}=0$ since $\left(W_{z(X)}\right)_{g}=0$. Consequently $\operatorname{sp}(W) \subseteq \operatorname{sp}(X)$.

Corollary 3.3. The bounded complex $\mathcal{L}^{++}$of an affine oriented matroid is pure, i.e., all the maximal chains in $\mathcal{L}^{++}$have the same length.

Proof. It follows from Theorem 3.2(2) that every maximal covector in $\mathcal{L}^{++}$has the same support. Therefore they all have the same rank in $\mathcal{L}$. Since $\mathcal{L}^{++} \cup\{0\}$ is an order ideal in $\mathcal{L}$, all the maximal chains in $\mathcal{L}^{++}$have the same length.

\section{Parallel Connection of Oriented Matroids}

The observant reader may have realized that Theorem 3.2(1) is a kind of direct sum decomposition. To make this more precise, we need to generalize the direct sum of hyperplane arrangements to affine oriented matroids. It turns out that the "direct sum" of affine oriented matroids is the operation know as parallel connection in matroid theory (see, e.g., [6]). ${ }^{1}$ In what follows we define parallel connection of oriented matroids and prove some basic properties. We make it a separate section since it is basic theory and should be of independent interest.

First we recall the direct sum of oriented matroids, which is a well-known and simple construction (see Proposition 7.6.1 of [2]). Let $\left(E_{1}, \mathcal{L}_{1}\right)$ and $\left(E_{2}, \mathcal{L}_{2}\right)$ be oriented matroids on disjoint ground sets. Their direct sum is the oriented matroid $(E, \mathcal{L})$ where $E=E_{1} \cup E_{2}$ and

$$
\mathcal{L}=\left\{X_{1} \cup X_{2}: X_{1} \in \mathcal{L}_{1} \text { and } X_{2} \in \mathcal{L}_{2}\right\} .
$$

The direct sum of affine oriented matroids (or parallel connection of oriented matroids) can be defined in a similar fashion. Recall that the restriction of a sign vector $X \in$ $\{+,-, 0\}^{E}$ to a subset $F \subseteq E$ is the sign vector $\left.X\right|_{F} \in\{+,-, 0\}^{F}$ defined by $\left(\left.X\right|_{F}\right)_{e}=$ $X_{e}$ for all $e \in F$.

Definition 4.1. Let $\left(E_{1}, \mathcal{L}_{1}\right)$ and $\left(E_{2}, \mathcal{L}_{2}\right)$ be two oriented matroids. Let $g_{1}$ and $g_{2}$ be nonloop elements in $E_{1}$ and $E_{2}$, respectively. We identify the two elements, $g:=g_{1}=g_{2}$, so that $E_{1} \cap E_{2}=g$. Let $E=E_{1} \cup E_{2}$ and

$$
\mathcal{L}=\left\{X \in\{+,-, 0\}^{E}:\left.X\right|_{E_{1}} \in \mathcal{L}_{1} \text { and }\left.X\right|_{E_{2}} \in \mathcal{L}_{2}\right\} .
$$

Then $(E, \mathcal{L})$ is an oriented matroid called the parallel connection of $\left(E_{1}, \mathcal{L}_{1}\right)$ and $\left(E_{2}, \mathcal{L}_{2}\right)$ along the base point $g$. When speaking of affine oriented matroids, we call $(E, \mathcal{L}, g)$ the direct sum of $\left(E_{1}, \mathcal{L}_{1}, g\right)$ and $\left(E_{2}, \mathcal{L}_{2}, g\right)$.

\footnotetext{
${ }^{1}$ The author thanks an anonymous referee for pointing this out.
} 
It is a routine exercise to verify that $\mathcal{L}$ satisfies the covector axioms in the above definition. Note that it is possible to define parallel connection even when $g$ is a loop. However, we choose to ignore the technical details here for simplicity. To make a distinction, we write $\oplus$ for the direct sum of oriented matroids and $\cup_{g}$ for the direct sum of affine oriented matroids. If the ground sets are understood, we sometimes write $\mathcal{L}=\mathcal{L}_{1} \cup_{g} \mathcal{L}_{2}$.

We provide the following proposition to show that our definition of the direct sum of affine oriented matroids is the right generalization of the direct sum of hyperplane arrangements. The proposition also justifies our use of the name parallel connection. Although this proposition is not essential to the rest of the paper, we believe it is of interest on its own. We refer the reader to [6] for all necessary definitions and facts related to matroids. Let $(E, \mathcal{L})$ be an oriented matroid and let $e \in E$. Recall that the contraction $(E, \mathcal{L}) / e$ is the oriented matroid on the ground set $E \backslash\{e\}$ with

$$
\mathcal{L} / e=\left\{X / e=\left.X\right|_{E \backslash\{e\}}: X \in \mathcal{L} \text { and } e \in z(X)\right\} \subseteq\{+,-, 0\}^{E \backslash\{e\}}
$$

being its set of covectors.

Proposition 4.2. Let $(E, \mathcal{L}, g)$ be an affine oriented matroid. Let $E_{1}$ and $E_{2}$ be subsets of $E$ such that $E_{1} \cup E_{2}=E$ and $E_{1} \cap E_{2}=\{g\}$. Let $\mathcal{L}_{i}=\left\{\left.X\right|_{E_{i}}: X \in \mathcal{L}\right\}$ for $i=1,2$. Then the following are equivalent:

(1) $(E, \mathcal{L}, g)=\left(E_{1}, \mathcal{L}_{1}, g\right) \cup_{g}\left(E_{2}, \mathcal{L}_{2}, g\right)$.

(2) The map $\varphi: \mathcal{L}^{+} \rightarrow \mathcal{L}_{1}^{+} \times \mathcal{L}_{2}^{+}$defined by $\varphi(X)=\left(\left.X\right|_{E_{1}},\left.X\right|_{E_{2}}\right)$ is an isomorphism of posets.

(3) $\operatorname{rank}\left(\mathcal{L}^{+}\right)=\operatorname{rank}\left(\mathcal{L}_{1}^{+}\right)+\operatorname{rank}\left(\mathcal{L}_{2}^{+}\right)$.

(4) $\operatorname{rank}(\mathcal{L} / g)=\operatorname{rank}\left(\mathcal{L}_{1} / g\right)+\operatorname{rank}\left(\mathcal{L}_{2} / g\right)$.

(5) $(E, \mathcal{L}) / g=\left(E_{1}, \mathcal{L}_{1}\right) / g \oplus\left(E_{2}, \mathcal{L}_{2}\right) / g$.

(6) Let $M, M_{1}$ and $M_{2}$ denote the underlying matroids of $\mathcal{L}, \mathcal{L}_{1}$ and $\mathcal{L}_{2}$, respectively. Then $M=P_{g}\left(M_{1}, M_{2}\right)$, the parallel connection of $M_{1}$ and $M_{2}$.

Proof. (1) $\Rightarrow$ (2) The map $\varphi$ is clearly injective and order preserving. The fact that $(E, \mathcal{L}, g)$ is the direct sum of $\left(E_{1}, \mathcal{L}_{1}, g\right)$ and $\left(E_{2}, \mathcal{L}_{2}, g\right)$ implies that $\varphi$ is also surjective.

(2) $\Rightarrow$ (3) $\Rightarrow$ (4) are trivial.

(4) $\Rightarrow$ (5) To simplify notation, we write $\mathcal{L}^{\prime}, \mathcal{L}_{1}^{\prime}$ and $\mathcal{L}_{2}^{\prime}$ to denote $\mathcal{L} / g, \mathcal{L}_{1} / g$ and $\mathcal{L}_{2} / g$, respectively. Suppose that $\operatorname{rank}\left(\mathcal{L}^{\prime}\right)=\operatorname{rank}\left(\mathcal{L}_{1}^{\prime}\right)+\operatorname{rank}\left(\mathcal{L}_{2}^{\prime}\right)$. We want to show that $\mathcal{L}^{\prime}=\mathcal{L}_{1}^{\prime} \oplus \mathcal{L}_{2}^{\prime}$. Since an oriented matroid is determined by its maximal covectors (topes), it suffices to show that $\mathcal{T}\left(\mathcal{L}^{\prime}\right)=\mathcal{T}\left(\mathcal{L}_{1}^{\prime} \oplus \mathcal{L}_{2}^{\prime}\right)$. The forward inclusion is clear. To prove the backward inclusion we use induction on $\operatorname{rank}\left(\mathcal{L}^{\prime}\right)$, the cases $\operatorname{rank}\left(\mathcal{L}^{\prime}\right) \leq 1$ being clear.

Let $X \in \mathcal{T}\left(\mathcal{L}_{1}^{\prime} \oplus \mathcal{L}_{2}^{\prime}\right)$ and let $Y$ be a covector in the direct sum that is covered by $X$. Then there is a nonloop element $e \in E \backslash\{g\}$ such that $Y_{e}=0$. Without loss of generality, say $e \in E_{1} \backslash\{g\}$. Then $\operatorname{rank}\left(\mathcal{L}^{\prime} / e\right)=\operatorname{rank}\left(\mathcal{L}^{\prime}\right)-1$ and $\operatorname{rank}\left(\mathcal{L}_{1}^{\prime}\right)=\operatorname{rank}\left(\mathcal{L}_{1}^{\prime} / e\right)-1$. Therefore $\operatorname{rank}\left(\mathcal{L}^{\prime} / e\right)=\operatorname{rank}\left(\mathcal{L}_{1}^{\prime} / e\right)+\operatorname{rank}\left(\mathcal{L}_{2}^{\prime}\right)$. Note that $Y / e$ is a tope in $\left(\mathcal{L}_{1}^{\prime} \oplus \mathcal{L}_{2}^{\prime}\right) / e=$ $\mathcal{L}_{1}^{\prime} / e \oplus \mathcal{L}_{2}^{\prime}$, so $Y / e \in \mathcal{L}^{\prime} / e$ by induction. Thus $Y \in \mathcal{L}^{\prime}$. Now let $Y_{1}, \ldots, Y_{k}$ be all the covectors that are covered by $X$ in $\mathcal{L}_{1}^{\prime} \oplus \mathcal{L}_{2}^{\prime}$. Then $Y_{i} \in \mathcal{L}^{\prime}$ for all $i=1, \ldots, k$. Hence $X=Y_{1} \circ \cdots \circ Y_{k} \in \mathcal{L}^{\prime}$. It is easy to see that $X$ must be maximal in $\mathcal{L}^{\prime}$.

(5) $\Rightarrow$ (1) Suppose that $\mathcal{L}^{\prime}=\mathcal{L}_{1}^{\prime} \oplus \mathcal{L}_{2}^{\prime}$, we want to show that $\mathcal{L}=\mathcal{L}_{1} \cup_{g} \mathcal{L}_{2}$. As before, it is enough to show that $\mathcal{T}(\mathcal{L})=\mathcal{T}\left(\mathcal{L}_{1} \cup_{g} \mathcal{L}_{2}\right)$. The forward inclusion is clear. 
To prove the backward inclusion we use induction on $\operatorname{rank}(\mathcal{L})$, the case $\operatorname{rank}(\mathcal{L})=1$ being clear.

Let $X \in \mathcal{T}\left(\mathcal{L}_{1} \cup_{g} \mathcal{L}_{2}\right)$ and $Y$ be a covector in the direct sum that is covered by $X$. Then there is a nonloop element $e \in E$ such that $Y_{e}=0$. Without loss of generality, say $e \in E_{1}$. If $e=g$, then $Y / e=Y / g \in\left(\mathcal{L}_{1} \cup_{g} \mathcal{L}_{2}\right) / g=\mathcal{L}_{1}^{\prime} \oplus \mathcal{L}_{2}^{\prime}=\mathcal{L}^{\prime}$. Therefore $Y \in \mathcal{L}$. If $e \neq g$, then $Y_{e} \in\left(\mathcal{L}_{1} \cup_{g} \mathcal{L}_{2}\right) / e=\mathcal{L}_{1} / e \cup_{g} \mathcal{L}_{2}$. Note that $\mathcal{L}^{\prime} / e=\left(\mathcal{L}_{1}^{\prime} \oplus \mathcal{L}_{2}^{\prime}\right) / e=\mathcal{L}_{1}^{\prime} / e \oplus \mathcal{L}_{2}^{\prime}$. Thus by induction $\mathcal{L} / e=\mathcal{L}_{1} / e \cup_{g} \mathcal{L}_{2}$. Therefore $Y_{e} \in \mathcal{L} / e$, and $Y \in \mathcal{L}$. Now let $Y_{1}, \ldots, Y_{k}$ be all the covectors that are covered by $X$ in $\mathcal{L}_{1} \cup_{g} \mathcal{L}_{2}$, then $Y_{i} \in \mathcal{L}$ for all $i=1, \ldots, k$. Hence $X=Y_{1} \circ \cdots \circ Y_{k} \in \mathcal{L}$. It is easy to see that $X$ must be maximal in $\mathcal{L}$.

(5) $\Rightarrow(6) \mathcal{L} / g=\mathcal{L}_{1} / g \oplus \mathcal{L}_{2} / g$ implies that $M / g=M_{1} / g \oplus M_{2} / g$. It then follows from Theorem 7.1.16(ii) of [6] that $M=P_{g}\left(M_{1}, M_{2}\right)$.

(6) $\Rightarrow$ (4) It follows from Theorem 7.1.15(i) of [6] that $\operatorname{rank}(M)=\operatorname{rank}\left(M_{1}\right)+$ $\operatorname{rank}\left(M_{2}\right)-1$. Since $g$ is not a loop, we get $\operatorname{rank}(M / g)=\operatorname{rank}\left(M_{1} / g\right)+\operatorname{rank}\left(M_{2} / g\right)$, or, equivalently, $\operatorname{rank}(\mathcal{L} / g)=\operatorname{rank}\left(\mathcal{L}_{1} / g\right)+\operatorname{rank}\left(\mathcal{L}_{2} / g\right)$.

Corollary 4.3. If $(E, \mathcal{L}, g)=\left(E_{1}, \mathcal{L}_{1}, g\right) \cup_{g}\left(E_{2}, \mathcal{L}_{2}, g\right)$ then $\mathcal{L}^{++} \cong \mathcal{L}_{1}^{++} \times \mathcal{L}_{2}^{++}$. An isomorphism is defined by the map $\varphi(X)=\left(\left.X\right|_{E_{1}},\left.X\right|_{E_{2}}\right)$ for all $X \in \mathcal{L}^{++}$.

Proof. Let $X \in \mathcal{L}^{+}$. By Proposition 4.2(2), it suffices to show that $X \in \mathcal{L}^{++}$if and only if $\left.X\right|_{E_{i}} \in \mathcal{L}_{i}^{++}$for $i=1,2$. If $X \notin \mathcal{L}^{++}$, then there exists $Y$ such that $0 \neq Y<X$ and $Y_{g}=0$. Either $\left.Y\right|_{E_{1}} \neq 0$ or $\left.Y\right|_{E_{2}} \neq 0$, say, $\left.Y\right|_{E_{1}} \neq 0$. Then $\left.Y\right|_{E_{1}}<\left.X\right|_{E_{1}}$ implies that $X_{E_{1}} \notin \mathcal{L}_{1}^{++}$. Conversely, if $\left.X\right|_{E_{i}} \notin \mathcal{L}_{i}^{++}$for some $i$, say $\left.X\right|_{E_{1}} \notin \mathcal{L}_{1}^{++}$, then there exists $Z$ such that $0 \neq Z<\left.X\right|_{E_{1}}$ and $Z_{g}=0$. Let $Y \in \mathcal{L}$ be the covector defined by $Y_{e}=Z_{e}$ if $e \in E_{1}$ and $Y_{e}=0$ otherwise. Then $\left.Y\right|_{E_{1}}=Z \in \mathcal{L}_{1}$ and $\left.Y\right|_{E_{2}}=0 \in \mathcal{L}_{2}$. Therefore $Y \in \mathcal{L}$. Now $Y<X$ implies that $X \notin \mathcal{L}^{++}$.

\section{A Central Decomposition Theorem}

In this section we reformulate Theorem 3.2 into a central decomposition theorem for affine oriented matroids. It is a direct generalization of Zaslavsky's central decomposition theorem for hyperplane arrangements.

Let $(E, \mathcal{L}, g)$ be an affine oriented matroid. Let $G \in\{+,-, 0\}^{E}$ be the sign vector defined by $G_{g}=+$ and $G_{e}=0$ if $e \neq g$. We say that $(E, \mathcal{L}, g)$ is central if $G \in \mathcal{L}$. Let $\mathcal{L} \backslash g=\left\{\left.X\right|_{E \backslash\{g\}}: X \in \mathcal{L}\right\}$. It is an easy exercise to show that $(E, \mathcal{L}, g)$ is central if and only if $(E, \mathcal{L})$ is the direct sum of the deletion $(E \backslash\{g\}, \mathcal{L} \backslash g)$ and the single-element oriented matroid $(\{g\},\{+,-, 0\})$. By Theorem 3.2(2), all the maximal covectors in the bounded complex $\mathcal{L}^{++}$have the same support, which will be denoted by $E_{1}$. Let $E_{2}^{\prime}=E \backslash E_{1}$ and $E_{2}=E_{2}^{\prime} \cup\{g\}$. Let $\mathcal{L}_{i}=\left\{\left.X\right|_{E_{i}}: X \in \mathcal{L}\right\}$ for $i=1,2$, and $\mathcal{L}_{2}^{\prime}=\left\{\left.X\right|_{E_{2}^{\prime}}: X \in \mathcal{L}\right\}$. We are now ready to state a central decomposition theorem.

\section{Theorem 5.1.}

(1) $(E, \mathcal{L}, g)=\left(E_{1}, \mathcal{L}_{1}, g\right) \cup_{g}\left(E_{2}, \mathcal{L}_{2}, g\right)$.

(2) $(E, \mathcal{L})=\left(E_{1}, \mathcal{L}_{1}\right) \oplus\left(E_{2}^{\prime}, \mathcal{L}_{2}^{\prime}\right)$, and $\left(E_{1}, \mathcal{L}_{1}\right)$ is the irreducible summand of $(E, \mathcal{L})$ containing $g$.

(3) $\mathcal{L}_{1}^{++} \cong \mathcal{L}^{++}$, and $\mathcal{L}_{2}^{++}$consists of a single covector whose support is $\{g\}$. 
(4) $\left(E_{2}, \mathcal{L}_{2}, g\right)$ is central. $(E, \mathcal{L}, g)$ is central if and only if $E_{2}=E$.

(5) $\left(E_{1}, \mathcal{L}_{1}, g\right)$ is not central, except when $E_{1}=\{g\}$.

(6) $E_{2}^{\prime}=\bigcap_{i=1}^{k} z\left(X_{i}\right)$, where $\left\{X_{1}, \ldots, X_{k}\right\}$ is the set of minimal covectors in $\mathcal{L}^{++}$.

(7) $\mathcal{L}_{1}=\mathcal{L} / E_{2}^{\prime}$.

Proof. (1) Let $X \in \mathcal{L}_{1} \cup_{g} \mathcal{L}_{2}$, so $\left.X\right|_{E_{1}} \in \mathcal{L}_{1}$ and $\left.X\right|_{E_{2}} \in \mathcal{L}_{2}$. Then there exist covectors $Y, Z \in \mathcal{L}$ so that $\left.X\right|_{E_{1}}=\left.Y\right|_{E_{1}}$ and $\left.X\right|_{E_{2}}=\left.Z\right|_{E_{2}}$. By Theorem 3.2(1), we have $Y_{E_{1}} \in \mathcal{L}$ and $Z_{E_{2}^{\prime}} \in \mathcal{L}$. Therefore $X=Y_{E_{1}} \circ Z_{E_{2}^{\prime}} \in \mathcal{L}$. This proves that $\mathcal{L}=\mathcal{L}_{1} \cup_{g} \mathcal{L}_{2}$.

(2) The proof of the fact that $\mathcal{L}=\mathcal{L}_{1} \oplus \mathcal{L}_{2}^{\prime}$ is almost identical to that of part (1). To prove that $\left(E_{1}, \mathcal{L}_{1}\right)$ is irreducible, we assume the contrary is true. Then $\left(E_{1}, \mathcal{L}_{1}\right)=$ $\left(E_{1}^{\prime}, \mathcal{L}_{1}^{\prime}\right) \oplus\left(E_{1}^{\prime \prime}, \mathcal{L}_{1}^{\prime \prime}\right)$ where $g \in E_{1}^{\prime}$ and $E_{1}^{\prime \prime} \neq \emptyset$. Let $X$ be a maximal covector in $\mathcal{L}^{++}$. Then $\operatorname{sp}(X)=E_{1}$. Therefore $X_{E_{1}^{\prime \prime}} \neq 0$ and $\left(X_{E_{1}^{\prime \prime}}\right)_{g}=0$. At the same time we have $\left.\left(X_{E_{1}^{\prime \prime}}\right)\right|_{E_{1}^{\prime}}=0 \in \mathcal{L}_{1}^{\prime},\left.\left(X_{E_{1}^{\prime \prime}}\right)\right|_{E_{1}^{\prime \prime}}=\left.X\right|_{E_{1}^{\prime \prime}} \in \mathcal{L}_{1}^{\prime \prime}$ and $\left.\left(X_{E_{1}^{\prime \prime}}\right)\right|_{E_{2}^{\prime}}=0 \in \mathcal{L}_{2}^{\prime}$. Hence $X_{E_{1}^{\prime \prime}} \in \mathcal{L}$. This contradicts the fact that $X \in \mathcal{L}^{++}$.

(3) This follows from Corollary 4.3.

(4) Let $X \in \mathcal{L}^{++}$. Then $\left.X\right|_{E_{2}} \in \mathcal{L}_{2},\left(\left.X\right|_{E_{2}}\right)_{g}=+$ and $\left(\left.X\right|_{E_{2}}\right)_{e}=0$ if $e \in E_{2} \backslash\{g\}$. This shows that $\left(E_{2}, \mathcal{L}_{2}, g\right)$ is central. If $E_{2}=E$, then $(E, \mathcal{L}, g)$ is also central. If $E_{2} \neq E$, assume that $(E, \mathcal{L}, g)$ is central, so $G \in \mathcal{L}$. Let $X$ be a maximal covector in $\mathcal{L}^{++}$. Then $\operatorname{sp}(X)=E_{1} \supset E \backslash E_{2}$, so there exists $e \neq g$ such that $X_{e} \neq 0$. Applying the Covector Axiom (L3) to $X$ and $-G$, we get a covector $Y$ with $Y_{g}=0$ and $Y_{e}=X_{e}$ otherwise. Now $0 \neq Y<X$ implies that $X \notin \mathcal{L}^{++}$, a contradiction to our choice of $X$. Therefore $(E, \mathcal{L}, g)$ is not central.

(5) If $E_{1}=\{g\}$ then $\left(E_{1}, \mathcal{L}_{1}, g\right)$ is trivially central. If $E_{1}$ contains elements other than $g$, then $\left(E_{1}, \mathcal{L}_{1}, g\right)$ is not central by the same proof as in part (4).

(6) Let $X$ be a maximal covector in $\mathcal{L}^{++}$. Then $E_{2}^{\prime}=z(X)$. Since $\widehat{\mathcal{L}}$ is atomic and $\mathcal{L}^{++} \cup\{0\}$ is an order ideal in $\widehat{\mathcal{L}}, X$ is the join of a subset of $\left\{X_{1}, \ldots X_{k}\right\}$. In particular, this shows that $\bigcap_{i=1}^{k} z\left(X_{i}\right) \subseteq z(X)$. On the other hand, let $e \in z(X)$. Then $e$ is in the zero set of every maximal covector in $\mathcal{L}^{++}$, since those covectors have the same support by Theorem 3.2(2). Therefore $e$ is in the zero set of every minimal covector in $\mathcal{L}^{++}$as well.

(7) This follows from (2).

\section{Collapsibility}

In this section we show that the bounded complex of an affine oriented matroid is collapsible. Let $\Gamma$ be a regular cell complex, and suppose that $\sigma \in \Gamma$ is a proper face of exactly one face $\tau \in \Gamma$. Then the complex $\Gamma^{\prime}=\Gamma \backslash\{\sigma, \tau\}$ is obtained from $\Gamma$ by an elementary collapse. Note that the condition on $\sigma$ and $\tau$ implies that $\tau$ is a maximal face of $\Gamma$ and $\sigma$ is a maximal proper face of $\tau$. If $\Gamma$ can be reduced to a single point by a sequence of elementary collapses, then $\Gamma$ is collapsible.

A convenient tool for showing that a complex is collapsible is provided by Forman's discrete Morse theory [4]. Here we follow the combinatorial description due to Chari [3]. Let $\Gamma$ be a regular cell complex. A matching $\mathbf{M}$ on $\Gamma$ is a collection of disjoint pairs of faces $(\sigma, \tau)$ such that $\sigma \lessdot \tau$ (so $\sigma$ is a maximal proper face of $\tau$ ). A closed path in $\mathbf{M}$ is a sequence $\left(\sigma_{0}, \tau_{0}\right), \ldots,\left(\sigma_{n-1}, \tau_{n-1}\right)$ of distinct pairs in the matching such that $\tau_{i} \gg \sigma_{i+1}$ 
for all $i \in \mathbb{Z} / n \mathbb{Z}$, where $n \geq 2$. An acyclic matching on $\Gamma$ is a matching that has no closed path in it. A critical cell of an acyclic matching $\mathbf{M}$ is a face that is unmatched in M. We only need the following special case of Forman's theory.

Lemma 6.1. Let $\Gamma$ be a regular cell complex. Then $\Gamma$ is collapsible if and only if there is an acyclic matching on $\Gamma$ whose only critical cell is a vertex.

Let $(E, \mathcal{L}, g)$ be an affine oriented matroid and let $\mathcal{L}^{++}$be its bounded complex. It is easy to see that $\mathcal{L}^{++}$is always nonempty-if $X$ is a minimal covector in $\mathcal{L}^{+}$then $X$ is an atom of $\mathcal{L}$, therefore $X \in \mathcal{L}^{++}$. In the realizable case this corresponds to the fact that the bounded complex of an essential hyperplane arrangement is nonempty.

To show that $\mathcal{L}^{++}$is collapsible, we use induction on $|E|$ to construct an acyclic matching on the covectors in $\mathcal{L}^{++}$. If $E=\{g\}$ then $\mathcal{L}^{++}$consists of a single covector, so we can take $\mathbf{M}$ to be the empty matching.

If $|E|>1$, take $h \in E \backslash\{g\}$. Consider the deletion $\left(E^{\prime}, \mathcal{L}^{\prime}, g\right)$ where $E^{\prime}=E \backslash\{h\}$ and $\mathcal{L}^{\prime}=\left.\mathcal{L}\right|_{E^{\prime}}$. By induction there is an acyclic matching $\mathbf{M}^{\prime}$ on $\left(\mathcal{L}^{\prime}\right)^{++}$such that the only critical cell is a vertex (a minimal covector in $\left(\mathcal{L}^{\prime}\right)^{++}$). We use $\mathbf{M}^{\prime}$ to construct an acyclic matching $\mathbf{M}$ on $\mathcal{L}^{++}$whose only critical cell is a vertex.

Let $H \in\{+,-, 0\}^{E}$ denote the sign vector with $H_{h}=+$ and $H_{e}=0$ if $e \neq h$. Let $\mathcal{H}=\left.\mathcal{L}\right|_{\{h\}}$. Then $\mathcal{L}=\mathcal{L}^{\prime} \oplus \mathcal{H}$ if and only if either $h$ is a loop or $H \in \mathcal{L}$. Because of Theorem 5.1(2) and (3), we may assume that $\mathcal{L}$ is irreducible. Therefore we assume that $h$ is not a loop and $H \notin \mathcal{L}$ from now on.

Let $A=\left\{Y \in \mathcal{L}^{++}:\left.Y\right|_{E^{\prime}} \notin\left(\mathcal{L}^{\prime}\right)^{++}\right\}$and $B=\mathcal{L}^{++} \backslash A$. We will construct a complete matching $\mathbf{M}_{A}$ on $A$ and a matching $\mathbf{M}_{B}$ on $B$. Then we will show that $\mathbf{M}=\mathbf{M}_{A} \cup \mathbf{M}_{B}$ is an acyclic matching on $\mathcal{L}^{++}$such that the only critical cell is a vertex.

Let $X$ be a sign vector in $\{+,-, 0\}^{E^{\prime}}$. We write $X s \in\{+,-, 0\}^{E}$ to denote the sign vector with $(X s)_{h}=s$ and $(X s)_{e}=X_{e}$ if $e \in E^{\prime}$.

Lemma 6.2. Let $X \in \mathcal{L}^{\prime}$. Then either $X s \in \mathcal{L}$ for a unique $s \in\{+,-, 0\}$, or $X s \in \mathcal{L}$ for all $s \in\{+,-, 0\}$.

Proof. If both $X+$ and $X-$ are in $\mathcal{L}$, then $S(X+, X-)=\{h\}$. Applying the Covector Axiom (L3), we get $X 0 \in \mathcal{L}$ as well. If both $X+$ and $X 0$ are in $\mathcal{L}$, then $X-=(X 0) \circ$ $(-(X+)) \in \mathcal{L}$. Similarly, if $X-, X 0 \in \mathcal{L}$ then $X+\in \mathcal{L}$ as well.

Lemma 6.3. Let $X \in \mathcal{L}^{\prime}$ such that $X s \in \mathcal{L}$ for a unique $s \in\{+,-, 0\}$. Let $[0, X]$ and $[0, X s]$ denote the corresponding intervals in $\mathcal{L}^{\prime}$ and $\mathcal{L}$, respectively. Then the map

$$
\varphi:[0, X s] \rightarrow[0, X], \quad \varphi(Y t)=Y
$$

is an isomorphism of posets.

Proof. The map $\varphi$ is clearly order-preserving and surjective. To show that it is injective, take $Y \in \mathcal{L}^{\prime}$ with $0 \leq Y \leq X$. If the preimage of $Y$ contains more than one element, then by the previous lemma $Y 0, Y+$ and $Y-$ are all covectors in $\mathcal{L}$. Thus $(X+)=$ 
$(Y+) \circ(X s) \in \mathcal{L}$ and $(X-)=(Y-) \circ(X s) \in \mathcal{L}$. This contradicts the uniqueness of $s$.

Corollary 6.4. Let $X \in \mathcal{L}^{\prime}$ such that $X s \in \mathcal{L}$ for a unique $s \in\{+,-, 0\}$. Then $X \in\left(\mathcal{L}^{\prime}\right)^{++}$if and only if $X s \in \mathcal{L}^{++}$.

Proof. The above lemma implies that $\mathcal{L}^{\prime}{ }^{\prime} \subseteq \widehat{\mathcal{L}}^{+}$if and only if $\mathcal{L}_{\leq X} \subseteq \widehat{\mathcal{L}}^{+}$. Consequently $X \in\left(\mathcal{L}^{\prime}\right)^{++}$if and only if $X s \in \mathcal{L}^{++}$.

Corollary 6.5. If $X t \in A$ for some $t \in\{+,-, 0\}$, then $X s \in \mathcal{L}$ for all $s \in\{+,-, 0\}$.

Lemma 6.6. Let $X \in\left(\mathcal{L}^{\prime}\right)^{+}$such that $X s \in \mathcal{L}$ for all $s \in\{+,-, 0\}$. If $X \notin\left(\mathcal{L}^{\prime}\right)^{++}$ then one of the following is true:

(1) $X s \notin \mathcal{L}^{++}$for all $s \in\{+,-, 0\}$;

(2) $X$ - and $X 0$ are in $\mathcal{L}^{++}$but $X+\notin \mathcal{L}^{++}$;

(3) $X+$ and $X 0$ are in $\mathcal{L}^{++}$but $X-\notin \mathcal{L}^{++}$.

Proof. If $X 0 \notin \mathcal{L}^{++}$then clearly $X s \notin \mathcal{L}^{++}$for all $s \in\{+,-, 0\}$. Now assume that $X 0 \in \mathcal{L}^{++}$. If neither $X+$ nor $X-$ is in $\mathcal{L}^{++}$, then there exist $Y s, Z t \in \mathcal{L} \backslash\{0\}$ such that $Y s<X+, Z t<X-$ and $Y_{g}=Z_{g}=0$. Note that we must have $s=+$, otherwise $Y s<X 0$ would contradict the assumption that $X 0 \in \mathcal{L}^{++}$. Similarly we have $t=-$. Recall our assumption that $H \notin \mathcal{L}$, so $Y \neq 0$. Since $S(Y+, Z-)=\{h\}$, applying the Covector Axiom (L3) we get $(Y \circ Z) 0 \in \mathcal{L}$. Then $(Y \circ Z)_{g}=0, Y \circ Z \neq 0$ and $(Y \circ Z) 0<X 0$. This is a contradiction to our assumption that $X 0 \in \mathcal{L}^{++}$. Hence at least one of $X+$ and $X-$ is in $\mathcal{L}^{++}$. It remains to show that they cannot both be in $\mathcal{L}^{++}$. Since $X \notin\left(\mathcal{L}^{\prime}\right)^{++}$, there exists $Y \in \mathcal{L}^{\prime} \backslash\{0\}$ such that $Y<X$ and $Y_{g}=0$. Now $Y t \in \mathcal{L}$ for some $t \in\{+,-, 0\}$. This implies that $X t \notin \mathcal{L}^{++}$, where $t=-$ or + .

The above lemma shows that the covectors in $A$ come in pairs. Therefore we have a complete matching

$$
\mathbf{M}_{A}=\{(X 0, X s): X 0, X s \in A \text { and } s \neq 0\} .
$$

Lemma 6.7. Let $X \in\left(\mathcal{L}^{\prime}\right)^{++}$. Then $X s \in \mathcal{L}$ implies that $X s \in \mathcal{L}^{++}$.

Proof. Assume that $X s \notin \mathcal{L}^{++}$. Then there exists $Y t \in \mathcal{L} \backslash\{0\}$ such that $Y t<X s$ and $Y_{g}=0$. Note that $Y<X$ since $0=Y_{g}<X_{g}=+$. If $Y \neq 0$ then we get a contradiction of the fact that $X \in\left(\mathcal{L}^{\prime}\right)^{++}$. If $Y=0$ then $t \neq 0$, which contradicts our assumption that $H \notin \mathcal{L}$.

Lemma 6.8. Let $X, Y \in \mathcal{L}^{\prime}$ such that $X \lessdot Y$ and $X 0, Y 0 \in \mathcal{L}$. If $Y s \in \mathcal{L}$ for some $s \in\{+,-\}$, then $X s \in \mathcal{L}$ as well. 
Proof. Without loss of generality, say $Y+\in \mathcal{L}$. Then $X 0 \lessdot Y 0 \lessdot Y+$ so the interval $[X 0, Y+]$ in $\mathcal{L}$ is of length 2 . Therefore it contains four elements (see Theorem 4.1.14 of [2]). The fourth element has to be $X+$.

We now construct a matching $\mathbf{M}_{B}$ on $B=\left\{X s \in \mathcal{L}^{++}: X \in\left(\mathcal{L}^{\prime}\right)^{++}\right\}$. Let $(X, Y)$ be a pair in the acyclic matching $\mathbf{M}^{\prime}$ on $\left(\mathcal{L}^{\prime}\right)^{++}$. Then $\mathbf{M}_{B}$ consists of pairs described in the following four cases:

Case 1: $X s \in \mathcal{L}$ for all $s \in\{+,-, 0\}$. In this case since $Y \in \mathcal{L}^{\prime}$, we have $Y t \in \mathcal{L}$ for some $t \in\{+,-, 0\}$. Then $Y+=(X+) \circ(Y t) \in \mathcal{L}$ and similarly $Y-\in \mathcal{L}$. Now Lemma 6.2 implies that $Y s \in \mathcal{L}$ for all $s$. Since $X, Y \in\left(\mathcal{L}^{\prime}\right)^{++}$, Lemma 6.7 then implies that $X s, Y s \in \mathcal{L}^{++}$for all $s$. Let $(X s, Y s) \in \mathbf{M}_{B}$ for all $s$.

Case 2: $X s \in \mathcal{L}$ for $s=+$ only. As in Case 1 we can show that $Y+\in \mathcal{L}$ in this case. Lemma 6.7 then implies that $X+, Y+\in \mathcal{L}^{++}$. Let $(X+, Y+) \in \mathbf{M}_{B}$. By Lemma 6.2 $Y 0 \in \mathcal{L}$ if and only if $Y-\in \mathcal{L}$. If they are both in $\mathcal{L}$ then they are in $\mathcal{L}^{++}$by Lemma 6.7. Let $(Y 0, Y-) \in \mathbf{M}_{B}$.

Case 3: $X s \in \mathcal{L}$ for $s=-$ only. This case is similar to Case 2.

Case 4: $X s \in \mathcal{L}$ for $s=0$ only. In this case if $Y s \in \mathcal{L}$ for all $s \in\{+,-, 0\}$ then we would have $X t \in \mathcal{L}$ for all $t \in\{+,-, 0\}$ by Lemma 6.8. That is a contradiction. Therefore $Y t \in \mathcal{L}$ for a unique $t \in\{+,-, 0\}$ by Lemma 6.2. Let $(X 0, Y t) \in \mathbf{M}_{B}$.

Let $X \in \mathcal{L}^{\prime}$ be the only critical cell of $\mathbf{M}^{\prime}$. Then the only unmatched cells in $\mathbf{M}=$ $\mathbf{M}_{A} \cup \mathbf{M}_{B}$ are those of the form $X s$ for some $s \in\{+,-, 0\}$.

Lemma 6.9. If $X$ is an atom in $\mathcal{L}^{\prime}$ then $X s \in \mathcal{L}$ for a unique $s \in\{+,-, 0\}$. Moreover $X s$ is an atom in $\mathcal{L}$.

Proof. Assume that $s$ is not unique. Then by Lemma $6.2 X s \in \mathcal{L}$ for all $s \in\{+,-, 0\}$. Take $e \in S(X+,-(X 0))=\operatorname{sp}(X)$ and apply the Covector Axiom (L3). We get a covector $Y+\in \mathcal{L}$ where $\operatorname{sp}(Y) \subseteq \operatorname{sp}(X) \backslash\{e\}$. Since $X$ is an atom this implies that $Y=0$. This is a contradiction to our assumption that $H \notin \mathcal{L}$.

It remains to show that $X s$ is an atom. Suppose that $Y t \in \mathcal{L}$ and $Y t<X s$. We must have $Y<X$ since $s$ is unique. Then $Y=0$ since $X$ is an atom. Finally $H \notin \mathcal{L}$ implies that $t=0$.

Since the critical cell $X$ of $\mathbf{M}^{\prime}$ is minimal in $\left(\mathcal{L}^{\prime}\right)^{++}$, it is necessarily an atom of $\mathcal{L}^{\prime}$. Therefore the above lemma together with Lemma 6.7 imply that $X s \in \mathcal{L}^{++}$for a unique $s \in\{+,-, 0\}$ and it is minimal in $\mathcal{L}^{++}$. Now we know that $X s$ is the only unmatched cell in $\mathbf{M}$ and it is a vertex.

It remains to show that $\mathbf{M}$ is acyclic. Assume this is not the case. Then there exists a closed path

$$
\left(X_{0} s_{0}, Y_{0} t_{0}\right),\left(X_{1} s_{1}, Y_{1} t_{1}\right), \ldots,\left(X_{n-1} s_{n-1}, Y_{n-1} t_{n-1}\right)
$$

in $\mathbf{M}$, where $Y_{i} t_{i} \gg X_{i+1} s_{i+1}$ for all $i \in \mathbb{Z} / n \mathbb{Z}$ and $n \geq 2$. We want to produce a closed path in $\mathbf{M}^{\prime}$, and therefore a contradiction. The following simple lemma will be useful. We omit its easy proof. 
Lemma 6.10. Let $X s, Y t \in \mathcal{L}$ such that $X s \lessdot Y$. Then either $X=Y, s=0$ and $t \in\{+,-\}$, or $X \lessdot Y$ in $\mathcal{L}^{\prime}$ and $s=t$.

If $t_{j}=0$ for some $j \in \mathbb{Z} / n \mathbb{Z}$ in our closed path, then clearly $s_{j}=0$. We claim that $t_{j-1}=0$ as well. By the above lemma it is enough to show that $Y_{j-1} \neq X_{j}$. Note that the pair $\left(X_{j} 0, Y_{j} 0\right)$ can only come from Case 1 or Case 4 of the matching $\mathbf{M}_{B}$. In particular, this means $\left(X_{j}, Y_{j}\right) \in \mathbf{M}^{\prime}$. If it is Case 4 , then $X_{j} s \in \mathcal{L}$ for $s=0$ only. Thus $Y_{j-1} \neq X_{j}$. If it is Case 1, then $X_{j} s$ must be the first element of a pair in $\mathbf{M}$ for every $s$. Thus it is impossible to have $Y_{j-1}=X_{j}$. Therefore $t_{j-1}=0$. It follows that $s_{i}=t_{i}=0$ and $\left(X_{i}, Y_{i}\right) \in \mathbf{M}^{\prime}$ for all $i \in \mathbb{Z} / n \mathbb{Z}$. Consequently we get a closed path $\left(X_{0}, Y_{0}\right), \ldots,\left(X_{n-1}, Y_{n-1}\right)$ in $\mathbf{M}^{\prime}$.

Now assume that $t_{i} \neq 0$ for all $i \in \mathbb{Z} / n \mathbb{Z}$. If $s_{i} \neq 0$ for all $i \in \mathbb{Z} / n \mathbb{Z}$ as well, then the above lemma forces $s_{i}=t_{i}=s$ for all $i \in \mathbb{Z} / n \mathbb{Z}$, where $s=+$ or - . It then follows from the construction of $\mathbf{M}$ that $\left(X_{i}, Y_{i}\right) \in \mathbf{M}^{\prime}$ for all $i \in \mathbb{Z} / n \mathbb{Z}$. Therefore $\left(X_{0}, Y_{0}\right), \ldots,\left(X_{n-1}, Y_{n-1}\right)$ is a closed path in $\mathbf{M}^{\prime}$.

Finally assume that $t_{i} \neq 0$ for all $i \in \mathbb{Z} / n \mathbb{Z}$, and $s_{j}=0$ for some $j \in \mathbb{Z} / n \mathbb{Z}$. Then by the above lemma we must have $Y_{j-1}=X_{j}=Y_{j}$. Since all three possible covectors of the form $X_{j} s$ have been used up, we necessarily have $X_{j-1} \lessdot Y_{j-1}=X_{j}$ and $X_{j}=Y_{j}>X_{j+1}$. Thus $s_{j-1}=t_{j-1}=: s$ and $t_{j}=s_{j+1}=t_{j+1}=: t$, where $s \neq t$. Hence, in particular, $n>2$. The three consecutive pairs in the closed path now look like

$$
\left(X_{j-1} s, X_{j} s\right),\left(X_{j} 0, X_{j} t\right),\left(X_{j+1} t, Y_{j+1} t\right) .
$$

Since these are disjoint pairs we have $X_{j} \neq Y_{j+1}$. It follows from the construction of $\mathbf{M}$ that $\left(X_{j-1}, X_{j}\right)$ and $\left(X_{j+1}, Y_{j+1}\right)$ are in $\mathbf{M}^{\prime}$. Therefore, after deleting trivial pairs of the form $\left(X_{j}, X_{j}\right)$ from the sequence $\left(X_{0}, Y_{0}\right), \ldots,\left(X_{n-1}, Y_{n-1}\right)$ we still have a closed path in $\mathbf{M}^{\prime}$ that contains at least two distinct pairs. This concludes our proof of the following theorem.

Theorem 6.11. Let $(E, \mathcal{L}, g)$ be an affine oriented matroid. Then its bounded complex $\mathcal{L}^{++}$is collapsible.

\section{Acknowledgement}

The author thanks an anonymous referee for many helpful suggestions.

\section{References}

1. A. Björner and G. M. Ziegler, Shellability of oriented matroids, Abstract, Workshop on Simplicial Complexes, IMA, University of Minnesota, March 1988; and Abstract, Conference on Ordered Sets, Oberwolfach, April 1988.

2. A. Björner, M. Las Vergnas, B. Sturmfels, N. White and G. Ziegler, Oriented Matroids, 2nd edn., Cambridge University Press, Cambridge, 1999.

3. M. Chari, On discrete Morse functions and combinatorial decompositions, Discrete Math., 217 (2000), 101-113.

4. R. Forman, Morse theory for cell complexes, Adv. Math. 134(1) (1998), 90-145. 
5. M. Las Vergnas, Matroïdes orientables, C. R. Acad. Sci. Paris Sér. A-B, 280 (1975), Ai, A61-A64.

6. J. Oxley, Matroid Theory, Oxford University Press, New York, 1992.

7. R. Stanley, An Introduction to Hyperplane Arrangements, Lecture notes, IAS/Park City Mathematics Institute, 2004.

8. T. Zaslavsky, Facing up to arrangements: face-count formulas for partitions of space by hyperplanes, Mem. Amer. Math. Soc. 1(1) (1975), no. 154.

9. G. M. Ziegler, The face lattice of hyperplane arrangements, Discrete Math., 74 (1988), 233-238.

Received January 3, 2005, and in revised form July 27, 2005. Online publication December 19, 2005. 